\title{
BMJ Open Characterising the gut microbiome in veterans with Gulf War Illness: a protocol for a longitudinal, prospective cohort study
}

\author{
Julie A Keating, ${ }^{1}$ Catherine Shaughnessy, ${ }^{2}$ Kelsey Baubie, ${ }^{\circ}{ }^{1}$ Ashley E Kates, ${ }^{1,2}$ \\ Nathan Putman-Buehler, ${ }^{2}$ Lauren Watson, ${ }^{2}$ Nadia Dominguez, ${ }^{2}$ Kal Watson, ${ }^{1}$ \\ Dane B Cook, ${ }^{1,3}$ David Rabago, ${ }^{4}$ Garret Suen, ${ }^{5}$ Ronald Gangnon, ${ }^{6}$ Nasia Safdar ${ }^{1,2}$
}

To cite: Keating JA, Shaughnessy C, Baubie K, et al. Characterising the gut microbiome in veterans with Gulf War IIIness: a protocol for a longitudinal, prospective cohort study. BMJ Open 2019;9:e031114. doi:10.1136/ bmjopen-2019-031114

- Prepublication history for this paper is available online. To view these files, please visit the journal online (http://dx.doi org/10.1136/bmjopen-2019031114).

Received 16 April 2019 Revised 16 May 2019 Accepted 17 May 2019
Check for updates

(C) Author(s) (or their employer(s)) 2019. Re-use permitted under CC BY-NC. No commercial re-use. See rights and permissions. Published by BMJ.

For numbered affiliations see end of article.

Correspondence to Dr Julie A Keating; julie.keating@va.gov

\section{ABSTRACT}

Introduction Approximately 25\%-35\% of the 1991 Gulf War Veteran population report symptoms consistent with Gulf War Illness (GWI), a chronic, multi-symptom illness characterised by fatigue, pain, irritable bowel syndrome and problems with cognitive function. GWI is a disabling problem for Gulf War Veterans, and there remains a critical need to identify innovative, novel therapies. Gut microbiota perturbation plays a key role in the symptomatology of other chronic multi-symptom illnesses, including myalgic encephalomyelitis/chronic fatigue syndrome (ME/CFS). Given similarities between ME/CFS and GWI and the presence of gastrointestinal disorders in GWI patients, Veterans with GWI may also have gut abnormalities like those seen with ME/ CFS. In this longitudinal cohort study, we are comparing the diversity (structure) and the metagenomes (function) of the gut microbiome between Gulf War Veterans with and without GWI. If we find differences in Veterans with GWI, the microbiome could be a target for therapeutic intervention to alleviate GWI symptoms.

Methods and analysis Participants answer questions about diet, exercise and lifestyle factors. Participants also complete a questionnaire (based on the Kansas case definition of GWI) regarding their medical history and symptoms; we use this questionnaire to group participants into GWI versus healthy control cohorts. We plan to enrol 52 deployed Gulf War Veterans: 26 with GWI and 26 healthy controls. Participants provide stool and saliva samples weekly for an 8-week period for microbiome analyses. Participants also provide blood samples at the beginning and end of this period, which we will use to compare measures of inflammation markers between the groups.

Ethics and dissemination The protocol was approved by the University of Wisconsin-Madison Health Sciences Institutional Review Board and the William S. Middleton Memorial Veterans Hospital Research and Development Committee. Results of this study will be submitted for publication in a peer-reviewed journal.

\section{INTRODUCTION}

Based on estimates by the Gulf War Research Advisory Committee and the 2010 Institute of Medicine report, approximately $25 \%-35 \%$ of
Strengths and limitations of this study

- This study will be among the first to evaluate the structure and functional characteristics of the gut microbiome in Gulf War Veterans with and without symptoms of Gulf War IIIness (GWI), advancing our understanding of the pathophysiology of GWI.

- Participants submit weekly stool and saliva samples for 8 weeks, and we compare (1) the alpha and beta diversity of the gastrointestinal microbiota and (2) the enrichment of genes through metagenomic analysis between Veterans with and without GWI.

- Participants also provide blood samples at weeks 1 and 8 to study markers of inflammation, which will provide additional insight into potential connections between gut microbiota and the immune system.

- GWI symptoms-which we use to group participants into control and GWI cohorts for comparisonare self-reported by participants using validated questionnaires.

the 1991 Gulf War Veteran population report symptoms consistent with Gulf War Illness (GWI). ${ }^{1-3}$ GWI is a chronic, multi-symptom illness characterised by fatigue, pain, irritable bowel syndrome and problems with cognitive function. GWI is a disabling problem for many Gulf War Veterans, and there have been only modest advances in the treatment for GWI. There remains a critical need for identification of innovative, novel therapies. ${ }^{4}$

Gut microbiota perturbation plays a key role in the symptomatology of chronic multisymptom illnesses similar to GWI, such as myalgic encephalomyelitis/chronic fatigue syndrome (ME/CFS). ${ }^{5-7}$ Although GWI and $\mathrm{ME} / \mathrm{CFS}$ are considered distinct conditions, both of them are characterised by similar complex, multi-organ chronic signs and symptoms. Moreover, Gulf War Veterans are more likely to meet the case definition for ME/CFS than non-deployed era Veterans. ${ }^{8}$ Given the 
overlap and similarity of symptoms, it is possible that gut microbiota abnormalities as described in ME/CFS may also be responsible for the symptomatology in GWI. ${ }^{9}$

Data suggest that individuals with GWI have an increased prevalence of gastrointestinal symptoms such as diarrhoea and abdominal pain and cramps, ${ }^{11}$ which may result in or be caused by intestinal dysbiosis (ie, alteration in the microbial community), low grade inflammation and immune activation. ${ }^{12}$ Given the body's largest immune reservoir resides in the gastrointestinal tract, interventions such as probiotics, dietary interventions or faecal microbiome transplants could favourably alter the gut microbiome, improve mucosal barrier function and decrease proinflammatory cytokines. Overall, interventions targeting the gut microbiome have the potential to positively influence not only gastrointestinal symptoms but also non-gastrointestinal symptoms in GWI Veterans. However, an understanding of the structure and function of the gut microbiome in Veterans with GWI is essential to the development of such interventions. Based on our review of the literature, the gut microbiome in Veterans with GWI and its relationship to the symptomatology of GWI have not yet been reported.

Here, we are characterising the gut microbiome of Gulf War Veterans with GWI compared with healthy Gulf War Veterans without GWI. This work is ongoing (2018-2020) and will be among the first to examine gut microbiome in Gulf War Veterans and will advance our understanding of the pathophysiology of GWI.

\section{STUDY AIMS AND HYPOTHESES}

In this longitudinal, prospective cohort study, we aim to compare the gut microbiomes between deployed Gulf War Veterans: 26 with GWI and 26 without GWI. Figure 1 provides an overview of the study methods. We hypothesise that the gut microbiome will be more diverse and will have a higher abundance of Bacteroidetes (rather than Firmicutes) in healthy Gulf War Veterans as compared with Veterans with GWI.

We also aim to evaluate the functional characteristics of the gut microbiome by comparing metagenomes between Gulf War Veterans with and without GWI. We hypothesise that metagenomes will differ in the enrichment of genes for predicted pathways between these groups. We will especially focus on microbiome pathways producing metabolites involved in symptoms frequently seen in GWI. For example, butyrate-a short-chain fatty acid metabolite produced by gut bacteria-has been associated with gastrointestinal symptoms. ${ }^{13}{ }^{14}$ Recent work in a mouse model of GWI has also shown that butyrate priming reduces metabolic abnormalities seen in GWI, for example, by restoring levels of gut tight junction proteins and reducing activation of a Toll-like receptor (TLR4; a mediator of inflammation). ${ }^{15}$

To test our hypotheses, we will extract, sequence and analyse genomic DNA from serial stool samples of Gulf War Veterans. We will compare the alpha and beta diversity

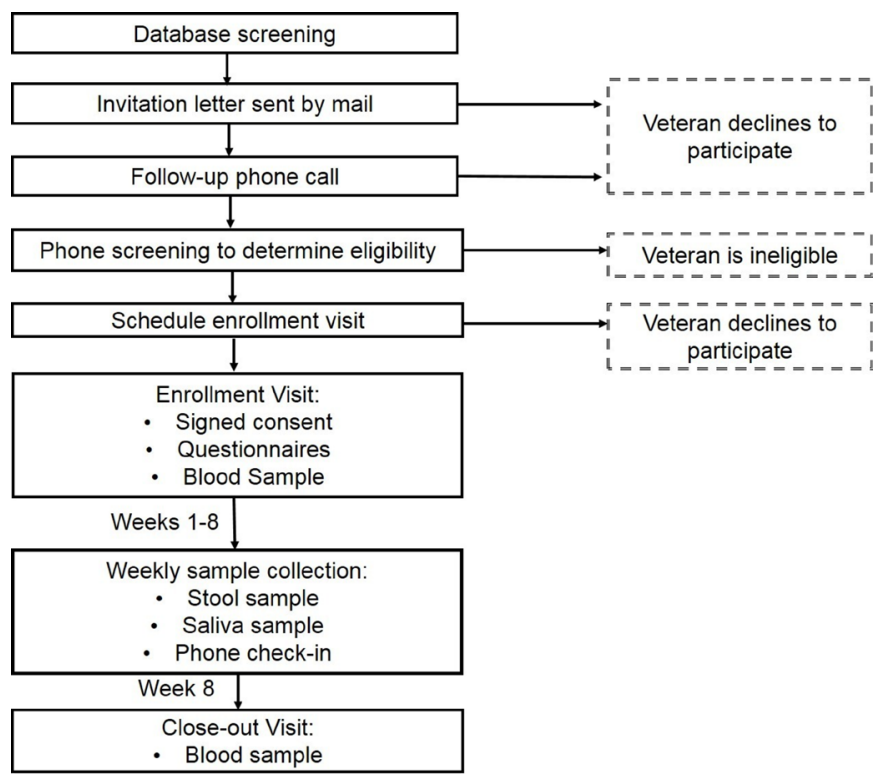

Figure 1 Potential participants are identified through a VA database screen of Veterans associated with the Madison VA who meet the inclusion criteria. Individuals interested in participating complete a screening by telephone, and those who are eligible and interested in participating attend an in-person enrolment visit. Participants provide weekly stool and saliva samples over an 8-week period and provide blood samples at Weeks 1 and 8.

of the microbiota between groups. We will also perform a shotgun metagenomics analysis on the collected samples.

To provide additional context to the gut microbiome results, we will also analyse the diversity of the oral microbiota through the collection and analysis of serial saliva samples. We will also collect blood samples to measure inflammation (C-reactive protein levels). We anticipate Veterans with GWI will have higher levels of C-reactive protein and inflammatory markers as compared with Gulf War Veterans without GWI.

\section{METHODS AND ANALYSIS}

\section{Recruitment and consent}

Participants are recruited primarily through invitation letters sent to local Gulf War Veterans identified from VA databases. Additional advertising strategies, including fliers, social media posts and local newspaper ads are also used. Veterans interested in participating complete a phone screen to determine eligibility based on the inclusion and exclusion criteria (boxes 1 and 2). Eligible Veterans are invited to an in-person enrolment session. At enrolment, written informed consent is obtained from participants by a trained member of the research team prior to beginning any further procedures. Participants receive $\$ 50$ after the enrolment visit and $\$ 50$ after the final visit for their participation in the study.

\section{Eligibility criteria and cohort assignment}

Inclusion and exclusion criteria for participants are listed in boxes 1 and 2. We are utilising a modified Kansas case 


\section{Box 1 Inclusion criteria for participants}

- Aged 43-75 years.

- Deployed to Persian Gulf as part of Operations Desert Shield and/or Desert Storm during the first Gulf War (1990-1991).

definition to determine eligibility and cohort assignment for this study. ${ }^{16}$ Veterans are excluded from the study if they report active, serious medical/psychological diagnoses which account for GWI-like symptoms or prevent accurate GWI-like symptom reporting. Veterans with well-controlled (or in remission) symptoms and who have not been hospitalised due to these conditions up to 5 years prior to enrolment may be eligible to participate.

We also use the Kansas case definition to group participants into two cohorts: deployed Gulf War Veterans with GWI and deployed healthy Gulf War Veterans. During enrolment, participants use the Kansas case definition questionnaire to self-report the severity of symptoms they experience within the six GWI symptom domains: fatigue, neurological/cognitive/mood, pain, gastrointestinal, respiratory and skin. For each symptom, participants select whether the symptom has no, mild, moderate or severe impacts on their daily lives. To be grouped into the GWI cohort, participants must endorse one or more moderate to severe symptoms in three or more of the six domains. One of the domains with moderate/severe symptoms must be fatigue. Participants who do not meet these criteria are grouped into the healthy Gulf War Veteran control cohort.

\section{Enrolment}

During the enrolment visit, participants provide medical history and health and lifestyle factors which can impact

\section{Box 2 Exclusion criteria for participants}

Diagnosed with a neurological or musculoskeletal condition (lupus, multiple sclerosis, Parkinson's disease, amyotrophic lateral sclerosis).

- Diagnosed with inflammatory bowel disease.

- One or more GWI Kansas case definition exclusionary conditions: schizophrenia, active cancer treatment or presence of cognitive/ physical impairments following a stroke.

- Unstable psychiatric illness (defined as hospitalisation within the previous 1 year): depression, bipolar disorder, or post-traumatic stress disorder.

- Active/unstable illness (defined as hospitalisation within the previous 5 years): diabetes, heart disease, rheumatoid arthritis, seizure disorder, kidney disease or liver disease.

- Current cancer treatment.

- Chronic infectious disease lasting 6 months or longer requiring hospitalisation within the previous 1 year.

- Current involvement in another investigational trial.

Pregnancy.

- Use of any of the following within 90 days prior to screening: antibiotics, probiotics, immunomodulatory medications, faecal microbiota transplant. the microbiome. Participants complete an in-house assessment addressing living situation (eg, type of home, rural/urban), health issues (eg, recent stays in long-term care facilities), oral health practices, current medication use and use of alcohol and tobacco. The participants also complete validated instruments: Holmes-Rahe Stress Inventory, ${ }^{17}$ Multidimensional Fatigue Inventory (MFI20), ${ }^{18} 19$ International Physical Activity Questionnaireshort form ${ }^{20}$ and National Cancer Institute's Diet History Questionnaire III. ${ }^{21}$

Participants report on branch of service during their Gulf War deployment and chemical, biological and pharmaceutical exposures from their deployment using an in-house questionnaire based on the VA list of Gulf War exposures. ${ }^{22}$ Participants also complete the Kansas case definition assessment as described earlier.

\section{Sample collection}

\section{Stool and saliva samples}

Participants are provided with collection kits, shipping materials and instructions to self-collect and mail their stool and saliva samples. Stool sample collection kits include a pair of clean gloves, stool collection tub, wooden tongue depressor, biohazard bag and pre-labelled sterile specimen cup. Saliva sample collection kits include a CryoELITE Cryogenic Storage Vial (Wheaton) saliva collection tube and a biohazard bag. The stool and saliva collection containers do not contain preservative agents. The participants are provided with ice packs and an insulated shipping container.

Participants collect at least five tablespoons of stool and $1-3.5 \mathrm{~mL}$ of saliva samples at their convenience once per week for 8 weeks and mail samples to the study team in the supplied shipping containers.

Participants have a window of 5 days each week to collect samples. The participant records the date and time of collection on the label provided for each sample and refrigerates the samples on collection (no freezing). Within 24hours of collection, the participant ships the samples overnight on completely frozen ice packs. Samples received more than 72 hours after the date noted on the sample label are rejected and are not included in microbiome analyses.

A member of the study team conducts a weekly phone check-in with each participant to ensure timely sample collection and to ask about any antibiotic/probiotic use or gastrointestinal symptoms over the week. This information is noted on study case report forms.

\section{Blood samples}

Two whole blood samples are drawn by venipuncture at enrolment and at the 8-week point: one sample is drawn into a $2 \mathrm{~mL}$ sodium heparin Vacuette (454302, Greiner Bio-one) and one sample is drawn into a $4 \mathrm{~mL}$ Vacuette containing potassium ethylenediaminetetraacetic acid (EDTA) (454021, Greiner Bio-one). Whole blood drawn in sodium heparin Vacuettes are processed immediately on collection for phagocytosis assay. Whole blood from 
the EDTA Vacuettes are centrifuged at $1690 \times \mathrm{g}$ for $15 \mathrm{~min}$ at room temperature immediately on collection, after which plasma is aspirated and aliquoted into cryovials to be stored at $-80^{\circ} \mathrm{C}$.

\section{Microbiome analyses}

The microbiome analyses methods follow the standard protocol developed by our research group, as previously published. ${ }^{23}$ Both 16S rRNA-targeted amplicon sequencing of the $\mathrm{V} 4$ region and shotgun metagenomics will be performed on the stool and saliva samples to address the aims of this project. 16S rRNA sequencing will be done on an Illumina MiSeq. A subset of the samples will be analysed using shotgun metagenomics on an Illumina HiSeq. All sequencing will be carried out at the University of Wisconsin-Madison Biotechnology Center. DNA extraction methods for each sample type are described below. For 16S rRNA sequencing, purified DNA is normalised to $5 \mathrm{ng} / \mu \mathrm{L}$, amplified using PCR with barcoded primers for the $16 \mathrm{~S} \mathrm{~V} 4$ region and sequenced using $2 \times 250 \mathrm{nt}$ reads.

\section{Stool total genomic DNA extraction}

$0.8-0.1 \mathrm{~g}$ of stool is added to a bead-beat tube containing approximately $300 \mathrm{mg}$ of $1.0 \mathrm{~mm}$ diameter zirconia/silica beads and vortexed to homogenise the stool. The sample is then centrifuged for $15 \mathrm{~min}$ at $4^{\circ} \mathrm{C}$ at $500 \times \mathrm{g}$. A total of $800 \mu \mathrm{L}$ of $2 \times$ Sodium Chloride-Tris-EDTA (STE) buffer is added to the supernatant and up to $1000 \mu \mathrm{L}$ is transferred to a new bead-beat tube containing $0.1 \mathrm{~mm}$ diameter zirconia/silica beads and one $4 \mathrm{~mm}$ stainless steel bead. For chemical lysis, $115 \mu \mathrm{L}$ of an enzymatic cocktail containing $50 \mu \mathrm{L}$ lysozyme $(10 \mathrm{mg} / \mathrm{mL}), 10 \mu \mathrm{L}$ mutanolysin $(1 \mathrm{mg} / \mathrm{mL}), 5 \mu \mathrm{L}$ lysostaphin $(5 \mathrm{mg} / \mathrm{mL})$ and $50 \mu \mathrm{L}$ $20 \%$ sodium dodecyl sulfate is added to each tube. Additionally, $700 \mu \mathrm{L}$ phenol:chloroform:isoamyl alcohol is added to the sample. Bead-beat tubes are then vortexed and incubated at $56^{\circ} \mathrm{C}$ for $30 \mathrm{~min}$. For mechanical lysis, bead-beat tubes are vortexed, placed in a Mini-BeadBeater-24 (Cat 112011, Biospec Products, Bartlesville, Oklahoma, USA) and beat for $3 \mathrm{~min}$. Tubes are centrifuged at $16000 \mathrm{xg}$ for $10 \mathrm{~min}$ at $4^{\circ} \mathrm{C}$. The top aqueous layer is transferred to a clean $2 \mathrm{~mL}$ tube, washed with an additional $500 \mu \mathrm{L}$ phenol:chloroform:isoamyl alcohol and vortexed. The sample is then centrifuged at $16000 \times \mathrm{g}$ for $10 \mathrm{~min}$ at $4^{\circ} \mathrm{C}$. The phenol:chloroform:isoamyl alcohol wash is repeated between 2 and 10 times to remove impurities from the sample until the aqueous layer is clean. The top aqueous layer is then transferred to a clean $2 \mathrm{~mL}$ microcentrifuge tube containing $70 \mu \mathrm{L}$ of $3 \mathrm{M}$ sodium acetate and $700 \mu \mathrm{L}$ isopropanol. The samples are inverted several times and subsequently incubated at $-20^{\circ} \mathrm{C}$ for $30 \mathrm{~min}$ to 1 hour. Each sample is centrifuged at $16000 \times \mathrm{g}$ $\left(4^{\circ} \mathrm{C}\right)$ for $20 \mathrm{~min}$ to collect the DNA pellet, which is then washed with $500 \mu \mathrm{L}$ cold $70 \%$ ethanol. The ethanol wash is repeated, and sample DNA pellets are dried for $5 \mathrm{~min}$ using a Savant SpeedVac (DNA120-230, Thermo Scientific, Waltham, Massachusetts, USA). Finally, dried DNA pellets are resuspended in $100 \mu \mathrm{L}$ TE buffer and stored overnight at $4^{\circ} \mathrm{C}$ or at $37^{\circ} \mathrm{C}$ for 1 hour to dissolve the DNA pellet. Samples are then purified using NucleoSpin Gel and PCR cleanup kit according to manufacturer's directions (Macherey-Nagel, Germany) and eluted in $40 \mu \mathrm{L}$ TE buffer. DNA is quantified using PicoGreen in a microplate reader (BioTek Instruments) and stored long term at $-80^{\circ} \mathrm{C}$.

\section{Saliva total genomic DNA extraction}

$1 \times$ PBS is added to the saliva tube to reach a volume of $1 \mathrm{~mL}$ which was then transferred to a bead-beat tube containing $0.1 \mathrm{~mm}$ diameter zirconia/silica beads and one $4 \mathrm{~mm}$ stainless steel bead. For chemical lysis, $25 \mu \mathrm{L}$ of an enzymatic cocktail containing $5 \mu \mathrm{L}$ lysozyme $(10 \mathrm{mg} /$ $\mathrm{mL}), 15 \mu \mathrm{L}$ mutanolysin $(1 \mathrm{mg} / \mathrm{mL}), 5 \mu \mathrm{L}$ lysostaphin $(5 \mathrm{mg} / \mathrm{mL})$ is added to each sample, vortexed and incubated at $37^{\circ} \mathrm{C}$ for $30 \mathrm{~min}$. Following incubation, $60 \mu \mathrm{L}$ of an enzymatic cocktail containing $10 \mu \mathrm{L}$ Proteinase $\mathrm{K}$ $(20 \mathrm{mg} / \mathrm{mL})$ and $50 \mu \mathrm{L} 10 \%$ sodium dodecyl sulfate is added to each tube for a second chemical lysis step. The samples are then vortexed and incubated at $55^{\circ} \mathrm{C}$ for $45 \mathrm{~min}$. Mechanical lysis is carried out by bead-beating the samples on high on a Mini-BeadBeater-24 (Cat 112011, Biospec Products) for $3 \mathrm{~min}$. Samples are centrifuged at $16000 \times \mathrm{g}$ for $3 \mathrm{~min}$ at $4^{\circ} \mathrm{C}$. The top aqueous layer is transferred to a sterile $2 \mathrm{~mL}$ tube and $0.1 \mathrm{vol}$ of $3 \mathrm{M}$ sodium acetate was added along with $0.6 \mathrm{vol}$ isopropanol and mixed by inversion. The samples are incubated at $-20^{\circ} \mathrm{C}$ for $30 \mathrm{~min}$ to 1 hour. Each sample is centrifuged at 16 $000 \times g\left(4^{\circ} \mathrm{C}\right)$ for $20 \mathrm{~min}$ to collect the DNA pellet, which is then washed with $500 \mu \mathrm{L}$ cold $70 \%$ ethanol. The ethanol wash is repeated, and sample DNA pellets are dried for 5 min using a Savant SpeedVac (DNA120-230, Thermo Scientific). Finally, dried DNA pellets are resuspended in $100 \mu \mathrm{L}$ TE buffer and stored overnight at $4^{\circ} \mathrm{C}$ or at $37^{\circ} \mathrm{C}$ for 1 hour to dissolve the DNA pellet. Samples are then purified using NucleoSpin Gel and PCR cleanup kit according to manufacturer's directions (Macherey-Nagel) and eluted in $40 \mu \mathrm{L}$ TE buffer. DNA is quantified using PicoGreen in a microplate reader (BioTek Instruments) and stored long term at $-80^{\circ} \mathrm{C}$.

\section{Blood analyses}

\section{C-reactive protein analyses}

Human C-reactive protein is detected by analysis with a commercially available ELISA kit (Human C-Reactive Protein ELISA Kit, KHA0031, Invitrogen). In short, a whole blood sample collected in a $4 \mathrm{~mL}$ potassium EDTA vacutainer is centrifuged at $1690 \times \mathrm{g}$ for $15 \mathrm{~min}$. Plasma from the sample is aliquoted into three cryogenic vials and stored at $-80^{\circ}$ C. Samples are batch processed for C-reactive protein using a commercially available ELISA kit (Human C-Reactive Protein ELISA Kit, KHA0031, Invitrogen) and plates are read with a BioTek Synergy HT microplate reader using BioTek Gen5 V.2.09 software. 
Flow cytometry analyses

Results from flow cytometry acquisition are analysed using FlowJo V.10 software. Monocytes and granulocytes are initially gated via Side Scatter (SSC) versus Forward Scatter (FFC). Those populations are further analysed via SSC versus fluorescence (BLA-1) where the fluorescent populations were further gated. The gated fluorescent populations indicated activity resulting from the phagocytosis assay (pHrodo Green E. coli BioParticles Phagocytosis Kit, P35381, Invitrogen) where increased fluorescence is indicative of increased cell death. ThermoFisher Attune Next Flow Cytometers used to collect phagocytosis assay data are located at the University of Wisconsin Carbone Cancer Center Flow Lab. Gating of this population using SSC versus BLA-1 allows for the generation of a curve measuring counts against emission wavelength. Curves for each condition are averaged together; SDs are calculated and $p$ values comparison is computed.

\section{Sample size determination and statistical analysis}

Using data from other studies of the gut microbiome, including studies on ME/CFS, we performed simulation-based estimates of power using the micropower package in $\mathrm{R}^{24}$ A proposed sample size of 26 healthy Gulf War Veterans and 26 Veterans with GWI will provide $80 \%$ power to detect an effect size (omega-squared) of 0.042 for the weighted UNIFRAC using a PERMANOVA test. ${ }^{25}$ The detectable effect size is comparable with, and more conservative than, the effect sizes observed in few studies that exist of the gut microbiome in ME/ $\mathrm{CFS},{ }^{5626-28}$ including one by our research group in which we compared the gut microbiota of 10 patients with ME/ CFS to 10 healthy controls. ${ }^{29}$

For 16S rRNA sequencing, raw sequences will be processed using VSEARCH ${ }^{30}$ and mothur. ${ }^{31}$ Contigs will be compiled and low-quality reads will be removed. Chimeras and short reads will be removed using VSEARCH. The GreenGenes database ${ }^{32}$ will be used to assign operational taxonomic units to the genus level (97\% similarity) where possible. Alpha diversity will be assessed using the Shannon and Inverse Simpson's diversity indices and richness will be calculated using the Abundance-based Coverage Estimator (ACE) and Chao1 indices. Beta diversity will be assessed to provide insights into community structure.

Shotgun metagenomics analysis will be conducted on a subset of samples. Phylogenetic binning will be conducted to determine if there is enrichment for specific taxa between samples. Baseline annotations will then be determined using a variety of databases to reconstruct metabolic and physiological pathways. Enrichment analyses will be conducted between samples to determine if specific sets of genes are over-represented or under-represented. These data will then be correlated to the other contextual data collected from each individual. We will focus our analyses on known pathways predicted to be implicated in modulating differences between Veterans with and without GWI, such as increases in short-chain fatty acids like butyrate and acetate.

To account for covariates, mixed effects linear regression will be used to estimate mean species diversity as a function of disease status (GWI vs control) as well as contextual factors including age, dietary fibre intake, tobacco and alcohol use and living situation. Confounders such as branch of Gulf War service and self-reported Gulf War chemical and biological exposures will also be controlled for using mixed linear regression models. C-reactive protein levels will be compared between Gulf War Veterans with GWI and healthy Veterans using non-parametric tests. We expect to find that levels of inflammatory markers will be higher in Veterans with GWI than in the control group. ${ }^{33}$

\section{Patient and public involvement}

We have worked with Veteran representatives and stakeholders, including local Veterans Service Officers, to share the study to Veterans who may be interested in participating. We also solicited feedback from these stakeholders into our recruitment materials and strategies to ensure communications were appropriate for this Veteran population. We plan to disseminate results back to the Veteran community through venues such as Veteran conferences/ meetings, working with local Veteran Service Organizations and working with the Madison VA Patient Education Resource Center.

\section{DISCUSSION}

GWI is a disabling problem for many Gulf War Veterans. To date, there has been little information on the pathology of the disease. There are no scientifically validated, effective treatments for GWI, though there have been modest advances in GWI treatments. ${ }^{4}$ Thus, the need for innovative treatment strategies remains critical. The gut microbiome presents a novel target for investigation. The high prevalence of gastrointestinal dysfunction in Gulf War Veterans in the context of literature demonstrating intestinal dysbiosis in patients with ME/CFS, another complex condition, suggests that alterations in the gut bacteria could play a role in the pathophysiology of GWI. This study is among the first to examine the gut microbiome structure and function in Gulf War Veterans.

The brain-gut axis-biochemical signalling between the gastrointestinal tract and the central nervous system-is increasingly recognised as important to health. ${ }^{34-40}$ The gut microbiome is known to impact the production, expression and turnover of neurotransmitters such as serotonin. These neurotransmitters affect cognitive and functional nervous system processes such as behaviour, mood and fatigue. The microbiome also affects the intestinal barrier and tight junction integrity, and bacterial metabolites influence mucosal immune regulation. Previous studies have shown chemical exposures common to the Gulf War (eg, the anti-nerve agent pyridostigmine bromide) can be used to create a mouse 
model of GWI. ${ }^{154142}$ These mouse models of GWI show an altered gut microbiome and a connection between this altered microbiome and neuroinflammation, suggesting a role for the brain-gut axis in GWI. ${ }^{1542}$ Through analyses of inflammatory markers as well as the structure and function of the microbiome in Veterans with GWI, our study aims to improve our understanding of the brain-gut axis and the role of the microbiome in GWI in humans.

Preliminary data from studies in mice and humans with ME/CFS have found a potential benefit in the use of probiotics to ameliorate fatigue symptoms. ${ }^{43-46}$ Initial studies have also found improvement in ME/CFS symptoms through the use of faecal microbiota transplant ${ }^{47}$; additional studies are ongoing into the use of faecal microbiota transplant to treat ME/CFS symptoms.

If we find that the gut microbiome is altered in Veterans with GWI, then probiotics, dietary intervention or faecal microbiota transplant may represent low-cost, low-risk treatment options for GWI symptoms. At the conclusion of this study, we expect to be positioned to pursue a clinical trial to investigate interventions targeting the gut microbiome as a way to alleviate symptoms in Veterans with GWI.

\section{ETHICS AND DISSEMINATION}

The study protocol was approved on 12 January 2018 and this manuscript reports on the most updated version of the protocol approved on 29 August 2018. All participants are informed prior to enrolment that participation is completely voluntary, that they can withdraw from participation at any time and that their decision to participate or not will not affect their healthcare in any way.

On completion of the study, we will present the results at major scientific conferences and will publish the results in a peer-reviewed journal.

\section{Author affiliations}

${ }^{1}$ Research, William S Middleton Memorial Veterans Hospital, Madison, Wisconsin, USA

${ }^{2}$ Department of Medicine, School of Medicine and Public Health, University of Wisconsin Madison, Madison, Wisconsin, USA

${ }^{3}$ Department of Kinesiology, University of Wisconsin Madison, Madison, Wisconsin, USA

${ }^{4}$ Department of Family Medicine and Community Health, School of Medicine and Public Health, University of Wisconsin Madison, Madison, Wisconsin, USA

${ }^{5}$ Department of Bacteriology, College of Agricultural and Life Sciences, University of Wisconsin Madison, Madison, Wisconsin, USA

${ }^{6}$ Department of Biostatistics and Medical Informatics, School of Medicine and Public Health, University of Wisconsin Madison, Madison, Wisconsin, USA

Contributors NS, DBC, DR, GS and RG conceived of the study concept and design. JAK drafted the overall protocol, with critical input from CS and KB for recruitment, screening, enrolment and data collection. AEK drafted protocol sections for DNA extraction, microbiome analyses and statistical analyses; NP-B, LW and ND for blood analyses and KW for flow cytometry. All authors provided critical feedback and approved the final version of the manuscript.

Funding This work is supported by Pilot Project Award \#CX-001574 from the United States (U.S.) Department of Veterans Affairs Clinical Sciences Research and Development Service. AEK was supported by an NLM training grant to the Computation and Informatics in Biology and Medicine Training Program (NLM 5T15LM007359).
Disclaimer The views expressed in this article are those of the authors and do not represent the position or policy of the US Department of Veterans Affairs or the United States government.

Competing interests None declared.

Patient consent for publication Not required.

Ethics approval University of Wisconsin-Madison Health Sciences Institutional Review Board: IRB00000366 William S. Middleton Memorial Veterans Hospital Research \& Development Committee.

Provenance and peer review Not commissioned; peer reviewed.

Open access This is an open access article distributed in accordance with the Creative Commons Attribution Non Commercial (CC BY-NC 4.0) license, which permits others to distribute, remix, adapt, build upon this work non-commercially, and license their derivative works on different terms, provided the original work is properly cited, appropriate credit is given, any changes made indicated, and the use is non-commercial. See: http://creativecommons.org/licenses/by-nc/4.0/.

\section{REFERENCES}

1. Fukuda K, Nisenbaum R, Stewart G, et al. Chronic multisymptom illness affecting Air Force veterans of the Gulf War. JAMA 1998;280:981-8.

2. Gronseth GS. Gulf war syndrome: a toxic exposure? A systematic review. Neurol Clin 2005;23:523-40.

3. Smith BN, Wang JM, Vogt D, et al. Gulf war illness: symptomatology among veterans 10 years after deployment. J Occup Environ Med 2013;55:104-10.

4. Golomb BA, Allison M, Koperski S, et al. Coenzyme Q10 benefits symptoms in Gulf War veterans: results of a randomized double-blind study. Neural Comput 2014;26:2594-651.

5. Frémont M, Coomans D, Massart S, et al. High-throughput $16 \mathrm{~S}$ rRNA gene sequencing reveals alterations of intestinal microbiota in myalgic encephalomyelitis/chronic fatigue syndrome patients. Anaerobe 2013;22:50-6.

6. Lakhan SE, Kirchgessner A. Gut inflammation in chronic fatigue syndrome. Nutr Metab 2010;7:79.

7. Maes M, Twisk FN, Kubera M, et al. Increased IgA responses to the LPS of commensal bacteria is associated with inflammation and activation of cell-mediated immunity in chronic fatigue syndrome. $J$ Affect Disord 2012;136:909-17.

8. Eisen SA, Kang HK, Murphy FM, et al. Gulf War veterans' health: medical evaluation of a U.S. cohort. Ann Intern Med 2005;142:881-90.

9. Ismail K, Kent K, Sherwood R, et al. Chronic fatigue syndrome and related disorders in UK veterans of the Gulf War 1990-1991: results from a two-phase cohort study. Psychol Med 2008;38:953-61.

10. Li B, Mahan CM, Kang HK, et al. Longitudinal health study of US 1991 Gulf War veterans: changes in health status at 10-year followup. Am J Epidemiol 2011;174:761-8.

11. Maule AL, Janulewicz PA, Sullivan KA, et al. Meta-analysis of selfreported health symptoms in 1990-1991 Gulf War and Gulf War-era veterans. BMJ Open 2018;8:e016086.

12. Bernstein CN, Forbes JD. Gut microbiome in inflammatory bowel disease and other chronic immune-mediated inflammatory diseases. Inflamm Intest Dis 2017;2:116-23.

13. Sun M, Wu W, Liu Z, et al. Microbiota metabolite short chain fatty acids, GPCR, and inflammatory bowel diseases. J Gastroenterol 2017;52:1-8.

14. Pozuelo M, Panda S, Santiago A, et al. Reduction of butyrate- and methane-producing microorganisms in patients with Irritable bowel syndrome. Sci Rep 2015;5:12693.

15. Seth RK, Kimono D, Alhasson F, et al. Increased butyrate priming in the gut stalls microbiome associated-gastrointestinal inflammation and hepatic metabolic reprogramming in a mouse model of Gulf War Illness. Toxicol Appl Pharmacol 2018;350:64-77.

16. Steele L. Prevalence and patterns of Gulf War illness in Kansas veterans: association of symptoms with characteristics of person, place, and time of military service. Am J Epidemiol 2000;152:992-1002.

17. Holmes $\mathrm{TH}, \mathrm{Rahe} \mathrm{RH}$. The social readjustment rating scale. $J$ Psychosom Res 1967;11:213-8.

18. Smets EM, Garssen B, Bonke B, et al. The Multidimensional Fatigue Inventory (MFI) psychometric qualities of an instrument to assess fatigue. J Psychosom Res 1995;39:315-25.

19. Goligher EC, Pouchot J, Brant R, et al. Minimal clinically important difference for 7 measures of fatigue in patients with systemic lupus erythematosus. J Rheumatol 2008;35:635-42. 
20. Monda V, Villano I, Messina A, et al. Exercise modifies the gut microbiota with positive health effects. Oxid Med Cell Longev 2017;2017:1-8.

21. National Cancer Institute. DHQ III: diet history questionnaire III. https://epi.grants.cancer.gov/dhq3/

22. Gulf War Exposures. US department of veterans affairs. Gulf War Veterans' illnesses web site. https://www.publichealth.va.gov/ exposures/gulfwar/sources/index.asp (Accessed Sep 2017).

23. Eggers S, Malecki KM, Peppard P, et al. Wisconsin microbiome study, a cross-sectional investigation of dietary fibre, microbiome composition and antibiotic-resistant organisms: rationale and methods. BMJ Open 2018;8:e019450.

24. R Core Team. R: A language and environment for statistical computing: R Foundation for Statistical Computing, 2015.

25. Kelly BJ, Gross R, Bittinger K, et al. Power and sample-size estimation for microbiome studies using pairwise distances and PERMANOVA. Bioinformatics 2015;31:2461-8.

26. Giloteaux L, Goodrich JK, Walters WA, et al. Reduced diversity and altered composition of the gut microbiome in individuals with myalgic encephalomyelitis/chronic fatigue syndrome. Microbiome 2016;4:30.

27. Jackson ML, Butt $H$, Ball M, et al. Sleep quality and the treatment of intestinal microbiota imbalance in chronic fatigue syndrome: a pilot study. Sleep Sci 2015;8:124-33.

28. Navaneetharaja N, Griffiths $\mathrm{V}$, Wileman $\mathrm{T}$, et al. A role for the intestinal microbiota and virome in myalgic encephalomyelitis/chronic fatigue syndrome (ME/CFS)? J Clin Med 2016;5:55.

29. Shukla SK, Cook D, Meyer J, et al. Changes in gut and plasma microbiome following exercise challenge in myalgic encephalomyelitis/chronic fatigue syndrome (ME/CFS). PLoS One 2015;10:e0145453.

30. Rognes T, Flouri T, Nichols B, et al. VSEARCH: a versatile open source tool for metagenomics. PeerJ 2016;4:e2584.

31. DeSantis TZ, Hugenholtz P, Larsen N, et al. Greengenes, a chimerachecked 16S rRNA gene database and workbench compatible with ARB. Appl Environ Microbiol 2006;72:5069-72.

32. Schloss PD, Westcott SL, Ryabin T, et al. Introducing mothur: opensource, platform-independent, community-supported software for describing and comparing microbial communities. Appl Environ Microbiol 2009;75:7537-41.

33. Johnson GJ, Slater BC, Leis LA, et al. Blood biomarkers of chronic inflammation in Gulf War illness. PLoS One 2016;11:e0157855.
34. Borre YE, Moloney RD, Clarke G, et al. The impact of microbiota on brain and behavior: mechanisms \& therapeutic potential. Adv Exp Med Biol 2014;817:373-403.

35. Moloney RD, Desbonnet L, Clarke G, et al. The microbiome: stress, health and disease. Mamm Genome 2014;25:49-74.

36. Burokas A, Arboleya S, Moloney RD, et al. Targeting the microbiotagut-brain axis: prebiotics have anxiolytic and antidepressant-like effects and reverse the impact of chronic stress in mice. Biol Psychiatry 2017;82:472-87.

37. Grenham S, Clarke G, Cryan JF, et al. Brain-gut-microbe communication in health and disease. Front Physiol 2011;2:94.

38. Dinan TG, Cryan JF. The microbiome-gut-brain axis in health and disease. Gastroenterol Clin North Am 2017;46:77-89.

39. Kelly JR, Kennedy PJ, Cryan JF, et al. Breaking down the barriers: the gut microbiome, intestinal permeability and stress-related psychiatric disorders. Front Cell Neurosci 2015;9:392.

40. Kennedy PJ, Cryan JF, Dinan TG, et al. Kynurenine pathway metabolism and the microbiota-gut-brain axis. Neuropharmacology 2017;112:399-412.

41. O'Callaghan JP, Kelly KA, Locker AR, et al. Corticosterone primes the neuroinflammatory response to DFP in mice: potential animal model of Gulf War Illness. J Neurochem 2015;133:708-21.

42. Alhasson F, Das S, Seth $\mathrm{R}$, et al. Altered gut microbiome in a mouse model of Gulf War Illness causes neuroinflammation and intestinal injury via leaky gut and TLR4 activation. PLoS One 2017;12:e0172914.

43. Singh PK, Chopra K, Kuhad A, et al. Role of Lactobacillus acidophilus loaded floating beads in chronic fatigue syndrome: behavioral and biochemical evidences. Neurogastroenterol Motil 2012;24:366-e170

44. Logan AC, Venket Rao A, Irani D. Chronic fatigue syndrome: lactic acid bacteria may be of therapeutic value. Med Hypotheses 2003;60:915-23.

45. Rao AV, Bested AC, Beaulne TM, et al. A randomized, double-blind, placebo-controlled pilot study of a probiotic in emotional symptoms of chronic fatigue syndrome. Gut Pathog 2009;1:6.

46. Sullivan A, Nord CE, Evengård B. Effect of supplement with lacticacid producing bacteria on fatigue and physical activity in patients with chronic fatigue syndrome. Nutr J 2009;8:4.

47. Borody TJ, Nowak A, Finlayson S. The Gl microbiome and its role in chronic fatigue syndrome: a summary of bacteriotherapy. Journal of the Australasian College of Nutritional and Environmental Medicine 2012:31. 